
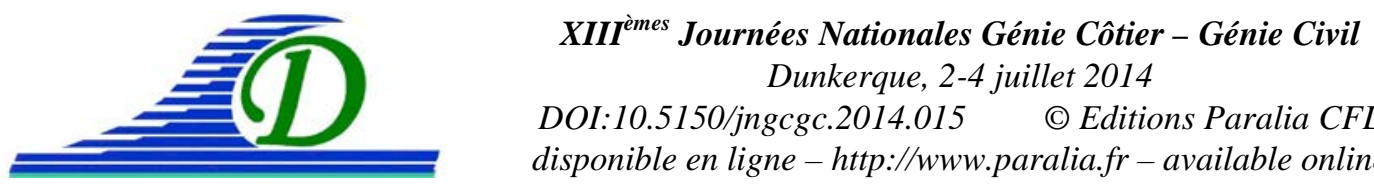

DOI:10.5150/jngcgc.2014.015 C Editions Paralia CFL

disponible en ligne - http://www.paralia.fr - available online

\title{
Hydrodynamique d'un écoulement induit par un rideau de bulles d'air : étude expérimentale
}

\author{
Djalal MERKOUNE ${ }^{1}$, Nizar ABCHA ${ }^{1}$, \\ Dominique MOUAZÉ ${ }^{1}$, Alexander EZERSKY ${ }^{1}$
}

\author{
1. Université de Caen, Faculté des Sciences, UMR 6143 CNRS - M2C, \\ Esplanade de la Paix, 14032 Caen, France. \\ Djalal.merkoune@gmail.com
}

\section{Résumé :}

Nous présentons les résultats expérimentaux, de l'influence d'un rideau de bulles d'air sur le déferlement des vagues, ce dernier est de type plongeant. Les expériences ont été réalisées en canal à houle à une profondeur d'eau constante. Une pompe à air a été utilisée pour générer les bulles d'air et contrôler leur concentration. Une faible concentration de bulles d'air provoque une dissipation d'énergie négligeable des vagues, cependant elle influence significativement la position du point de déferlement. A partir de cela, nous avons mené une étude expérimentale pour appréhender d'avantage les mécanismes physiques pilotant l'interaction bulles d'air/déferlement. La vitesse des bulles d'air a été mesurée à l'aide d'une sonde conductive à double pointe tandis que les champs de vorticité d'écoulement ont été caractérisés au moyen d'un vélocimètre ADV.

Mots-clés : Déferlement, Bulles d’air, Energie, Canal à houle, Interaction.

\section{Introduction}

Le déferlement des vagues joue un rôle important dans de nombreux processus en dynamique côtière. Ce phénomène est accompagné de formation de bulles, d'embruns et d'écume dont la recherche sur les mécanismes en cause est très active. (GRANT et al., 1998) ont effectué des expériences in-situ et ont ainsi décrit en détails la distribution de la taille des bulles d'air. MUKHERJEE \& KANDLIKAR (2007) ont enrichit ce travail en réalisant des simulations numériques sur un modèle de Navier-Stokes complet. En outre, CUMMINGS \& CHANSON (1997) ont dressé une revue détaillée sur les techniques d'entrainement de bulles d'air sous la surface de l'eau.

Plusieurs modélisations mathématiques et numériques décrivent et caractérisent les niveaux d'aération atteints dans les vagues. En particulier, l'analyse des étapes de l'entrainement d'air dans les vagues et les mesures d'évolution des poches d'air dans un écoulement constituent une problématique de premier ordre. (VASSILEV et al., 2007) ont caractérisé l'écoulement et la forme 2D des bulles d'air et ce par voie expérimentale et numérique. Entre autre, ils ont examiné l'évolution spatio-temporelle du mélange air/eau avec la prise en compte de l'effet de la viscosité sur le mouvement des bulles 
d'air. L'originalité de ce travail consiste à étudier l'influence d'une faible concentration de bulles d'air sur le déferlement des vagues.

\section{Dispositif expérimental}

\subsection{Canal à houle}

Le canal à houle utilisé est une structure vitrée de $22 \mathrm{~m}$ de longueur, 0,8 $\mathrm{m}$ de largeur et de $0,8 \mathrm{~m}$ de profondeur (figure 1). Il permet de simuler de nombreuses conditions de houles sur une hauteur maximale de $0,4 \mathrm{~m}$. Les parois transparentes du canal permettent de mettre en œuvre des techniques de mesure optique telles que la Vélocimétrie Doppler Laser, la visualisation par nappes laser ou encore la Vélocimétrie par Imagerie de Particules (PIV). Le fond est constitué d'une plaque PVC d'épaisseur $2 \mathrm{~cm}$, considérée ici comme un fond hydrauliquement lisse. L'ensemble de cette installation repose sur des poteaux métalliques de $0,5 \mathrm{~m}$ de haut. Les vagues sont générées à l'aide d’un batteur placé à l'extrémité du canal, contrôlé par un ordinateur. Un conduit cylindrique de longueur égale à la largeur du canal a été foré pour obtenir des trous de diamètre $\mathrm{d}=1 \mathrm{~mm}$ espacés d'une distance constante égale à $1 \mathrm{~cm}$. Ce dernier a été fixé sur le fond du canal et est relié à une pompe d'aération équipée d’un débitmètre.

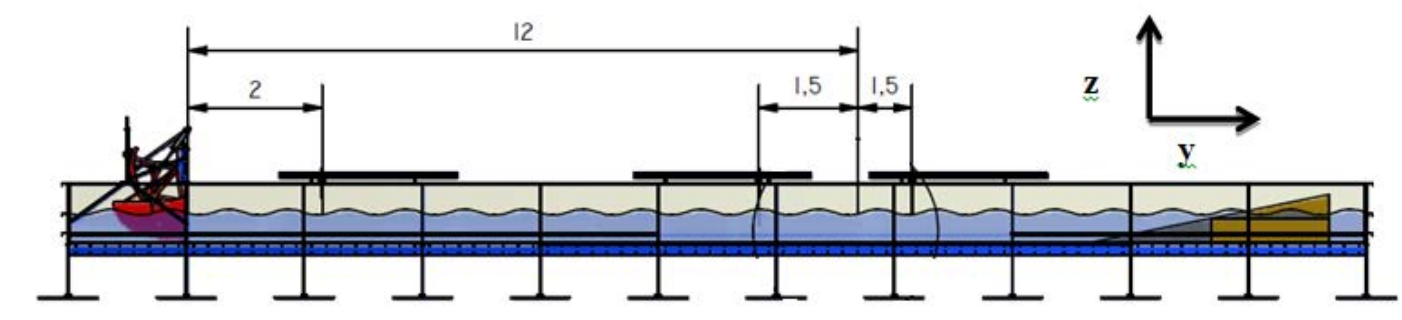

Figure 1. Plan du canal à houle avec un zoom sur la position du rideau de bulles situé entre deux sondes de surface libre (la cotation est en $\mathrm{m}$ ).

L'axe du tube est perpendiculaire à la paroi latérale du canal. L'axe "z" est pointé vers le haut et indique la hauteur au-dessus de la sortie des trous. L'axe "y" est horizontal, perpendiculaire à l'axe " $z$ " et est orienté dans le sens d’écoulement.

\subsection{Sonde conductive}

Les propriétés d'écoulement air-eau ont été mesurées avec une sonde conductive à double-pointe. La sonde est équipée de deux capteurs identiques.

La distance entre les extrémités des deux pics d'une pointe de la sonde est de $1 \mathrm{~mm}$, tandis que la distance de séparation entre les extrémités des deux pointes intérieures est de $3 \mathrm{~mm}$. 


\section{XIII ${ }^{\text {èmes }}$ Journées Nationales Génie Côtier - Génie Civil \\ Dunkerque, 2-4 juillet 2014}

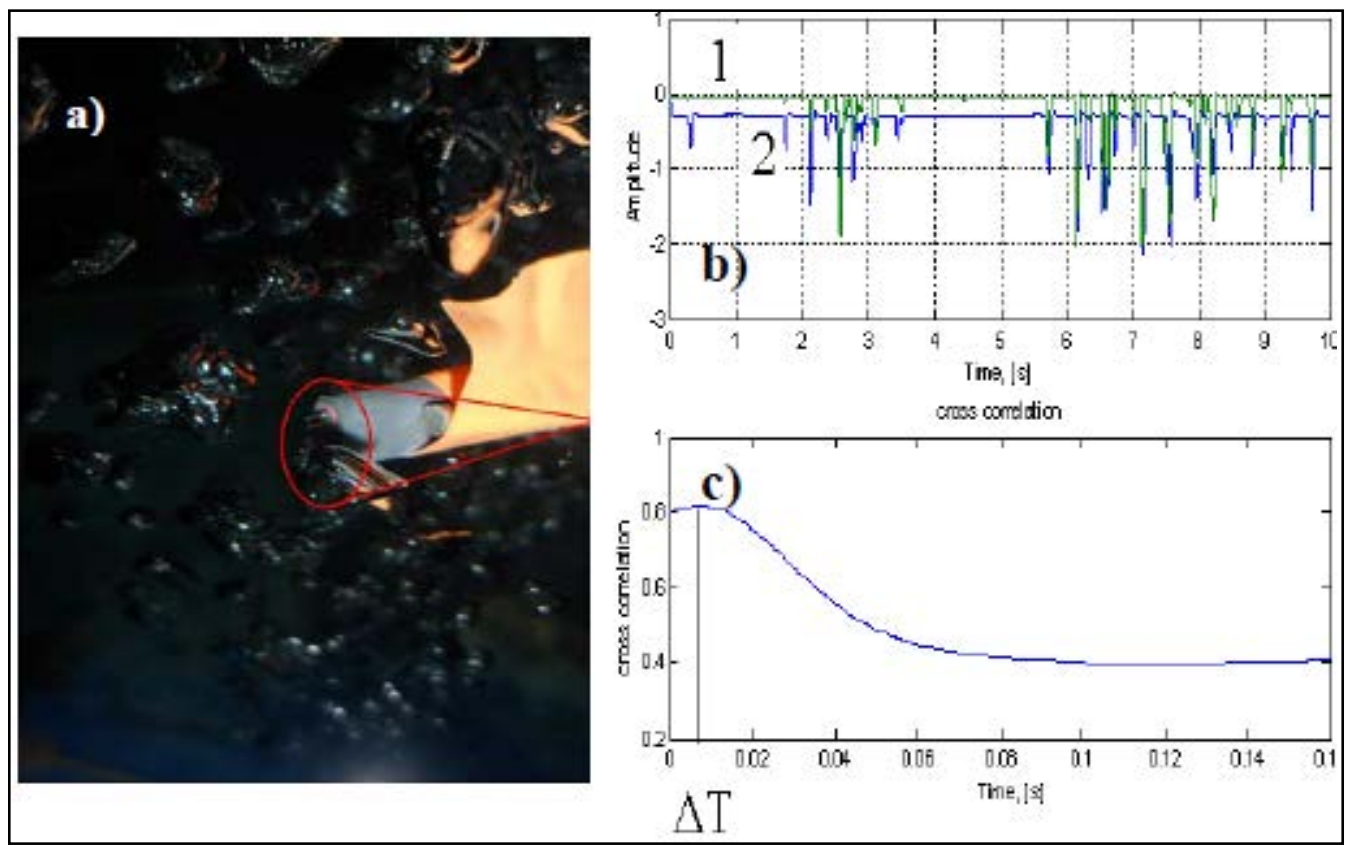

Figure 2. a) Image de la sonde conductive à double-pointe. b) Sorties temporelles des sondes 1.2. c) Corrélation croisée.

\section{Résultats}

\subsection{Déferlement des vagues en présence de bulles d'air}

Pour étudier l'influence des bulles d'air sur le processus déferlement, nous avons procédé de la manière suivante. Un rideau de bulles d'air a été généré au moyen d'une pompe d'aération et ce à une distance de $y_{0}=13 \mathrm{~m}$ du générateur de vagues. Pour comprendre la relation entre le déferlement et la quantité d'air disponible dans le mélange air/eau, l'expérience a été effectuée avec différents débits d'air. À l'aide d'une caméra rapide à haute résolution, nous avons suivi l'évolution de la surface libre dans l'espace et repéré la position du déferlement. La figure 3 illustre la position du déferlement plongeant par focalisation dispersive en fonction du débit d'air injecté. Nous constatons que cette position est très sensible même pour de faibles débits d'air. Le déplacement du point de déferlement $\left(y-y_{0}\right)$ vers le générateur de vagues atteint une valeur maximale de $50 \mathrm{~cm}$.

\subsection{La dissipation de l'énergie du train d'ondes en présence d'un rideau de bulles d'air}

Pour étudier l'influence des bulles sur le déferlement de façon plus précise, nous avons calculé les changements d'énergie pour différents flux d'air. La valeur de l'énergie du train d'ondes a été calculée suivant l'expression suivante :

$E=\frac{\rho g}{2} C_{g} \int_{0}^{T}<\eta-<\eta>>^{2} d t$ 

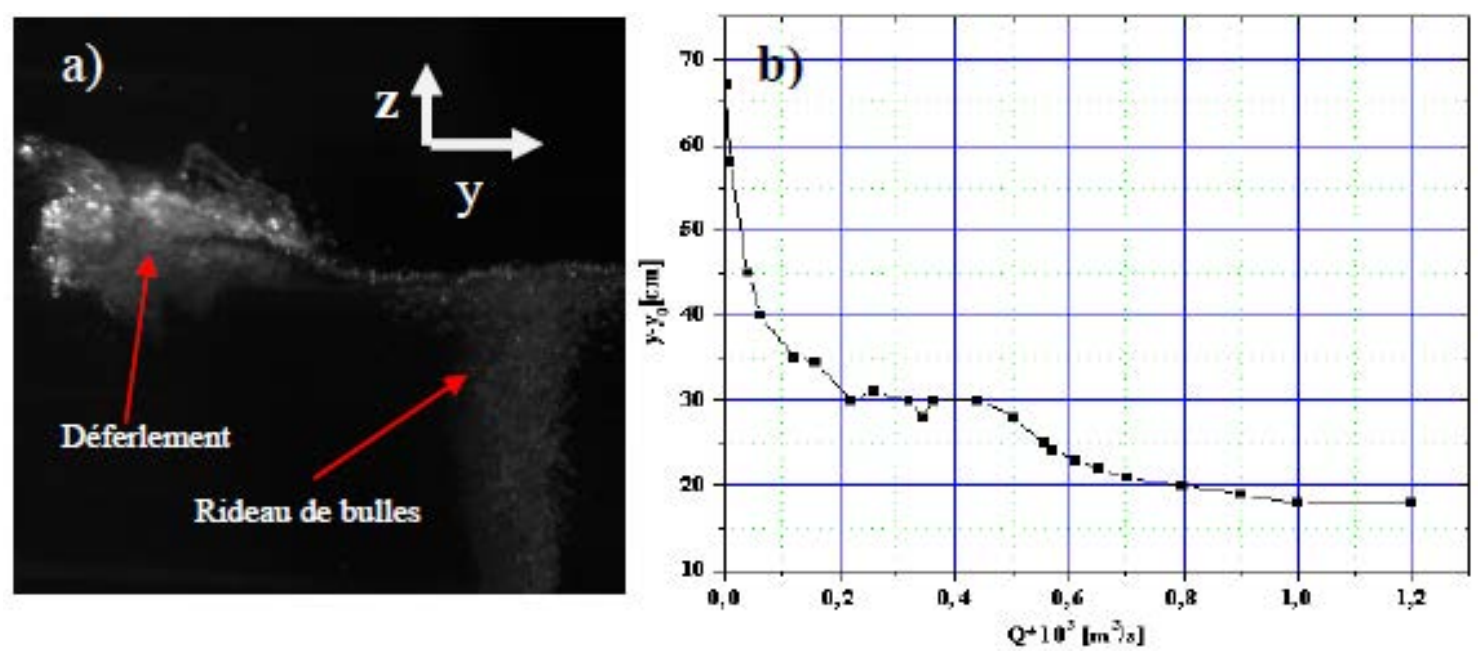

Figure 3. a) Photographie de l'expérience de déferlement des vagues en présence des bulles d'air, b) Abscisse du point de déferlement en fonction du débit d'air ( $\left.y_{0}=13 m\right)$.

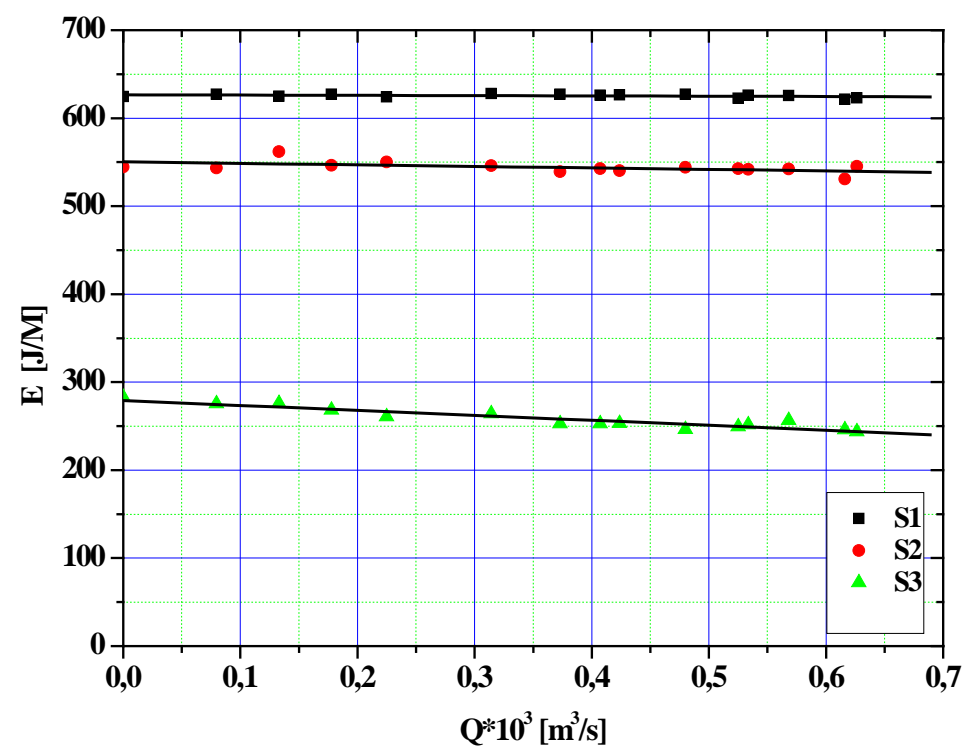

Figure 4. Energie du paquet d'ondes en présence d'un rideau de bulles d'air artificiel à différentes positions.

où $T=1.33 \mathrm{~s}$ est la période du paquet d'ondes, $\eta$ l'élévation de la surface libre en $\mathrm{m}$. $C_{g}$ la vitesse de groupe, et $g$ l'accélération de la pesanteur $\mathrm{m} / \mathrm{s}^{2}$. La figure 4 indique l'énergie du paquet d'ondes en fonction du débit d'air, avant et après l'interaction du paquet avec le rideau de bulles d'air. Sur ce graphe, nous remarquons qu'entre les deux premières sondes S1 et S2, une faible dissipation d'énergie de l'ordre de $12 \%$ est enregistrée. Cette perte d'énergie reste quasi-constante pour l'ensemble des valeurs de débit d'air. De cela, découle que cette perte d'énergie est le résultat du frottement avec le fond et les parois latéraux et de la dissipation visqueuse. En revanche, entre la première sonde $\mathrm{S} 1$ et la troisième $\mathrm{S} 3$, soit une distance de $11 \mathrm{~m}$, la perte d'énergie atteint 


\section{XIII ${ }^{\text {èmes }}$ Journées Nationales Génie Côtier - Génie Civil \\ Dunkerque, 2-4 juillet 2014}

une valeur de 50\% de l'énergie initiale. Cette dissipation est le résultat du déferlement glissant de la vague. Une petite quantité supplémentaire de dissipation d'énergie est observée après la génération de rideaux de bulles. Effectivement, cette quantité d'énergie dissipée est très faible- environ $10 \%$ de l'énergie initiale- contrairement à celle conditionnée par le déferlement. Ces résultats sont en bon accord avec ceux de (ZHANG et al., 2010), ils ont montré que le coefficient de transmission est de l'ordre de 95\% pour les mêmes conditions expérimentales que les nôtres.

\subsection{Caractéristiques cinématiques des bulles d'air}

En absence d'un train d'onde propagatif, la vitesse des bulles d'air croit de manière symétrique à l'axe du rideau. Le premier graphique représente l'évolution longitudinale des bulles d'air dans le canal pour un débit d'air de $Q=1,2 \times 10^{-3} \mathrm{~m}^{3} / \mathrm{s}$. (figure 5).

A partir du fond du canal, les bulles d'air atteignent des vitesses importantes de quelques dizaines de $\mathrm{cm} / \mathrm{s}$. Le profil des vitesses présente deux régimes : une accélération près du fond et une décélération près de la surface libre. Nous avons constaté que les bulles d'air ont tendance à s'orienter dans le sens de l'écoulement généré par le rideau durant leurs phases de croissance. Cette orientation est d'autant plus marquée quand le cisaillement des vitesses dans le liquide est important.

La trajectoire des bulles d'air s'oriente fortement vers l'horizontal. Nous avons enregistré des vitesses de bulles très proche de celles trouvées par (KULKARNI \& JOSHI, 2005). Ces derniers ont montré une évolution des vitesses verticales similaire à celle de notre étude : une zone d'augmentation de la vitesse verticale des bulles entre $0,1 \mathrm{~m} / \mathrm{s}$ à $0,4 \mathrm{~m} / \mathrm{s}$ pour des diamètres de bulles allant de $0.5 \mathrm{~mm}$ à $1 \mathrm{~mm}$, et une deuxième zone de décélération de $0,4 \mathrm{~m} / \mathrm{s}$ à $0,35 \mathrm{~m} / \mathrm{s}$ pour une augmentation du diamètre des bulles de $1 \mathrm{~mm}$ à $5 \mathrm{~mm}$. Ces valeurs de vitesses sont supérieures à celles trouvées par WICHTERLE et al. (2009) pour la vitesse d'une bulle isolée. Cette différence peut être attribuée aux effets de sillage des bulles relativement les unes aux autres (BYALKO, 2011). Dans le sens positive des "z", les bulles d'air s'accélèrent comme la poussée d'Archimède prédomine la force de gravité ; du fait de l'augmentation de la taille de la bulle d'air (diminution de la pression hydrostatique avec l'altitude dans la colonne d'eau), l'écoulement local autour de la bulle n'est plus décrit par l'équation de la vitesse limite de Stokes mais par celle d'Oseen (BRUNCOTTAN, 1986). Dans ce contexte, une diminution de la vitesse de montée des bulles avec l'augmentation de leurs tailles a été remarquée par (AMOL \& JYESHTHARAJ, 2005). Cela implique que la vitesse limite ascensionnelle des bulles est inférieure à ce qu'elle serait en écoulement local de Stokes. Ceci peut expliquer la diminution de la vitesse des bulles dans la deuxième zone. 


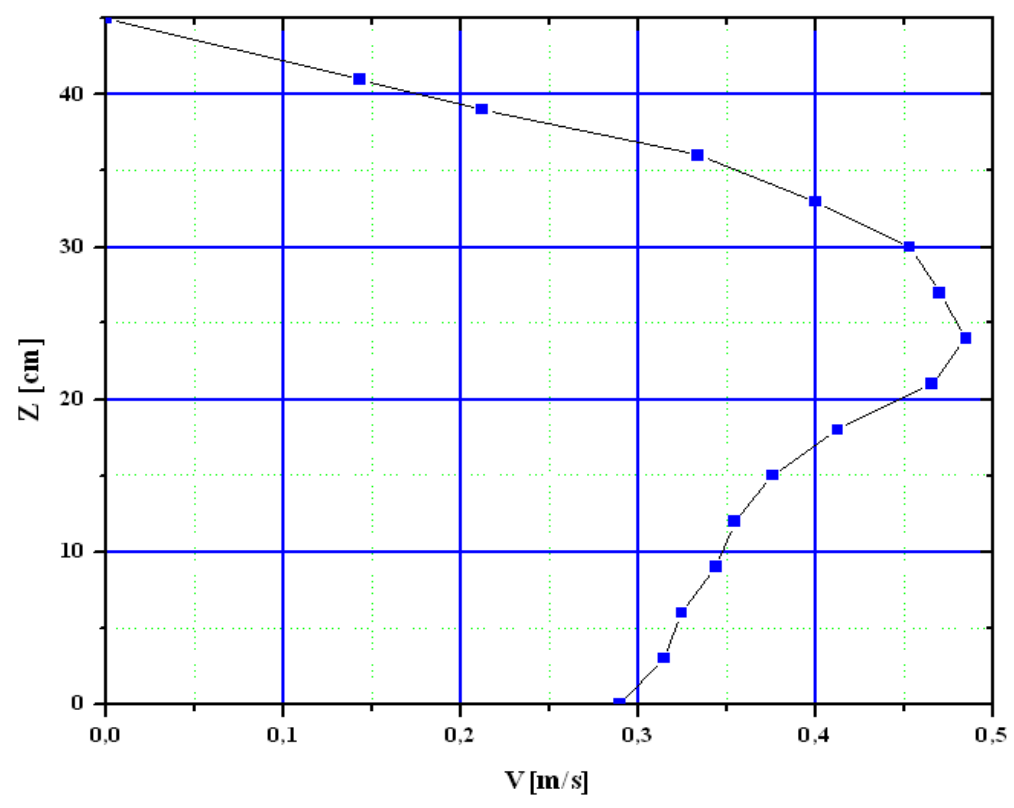

Figure 5. Evolution verticale de la vitesse des bulles d'air dans la colonne d'eau.

\subsection{Les caractéristiques hydrodynamiques du rideau de bulles d'air}

Dans cette partie nous avons étudié les mécanismes qui contrôlent l'hydrodynamique des bulles d'air durant l'écoulement. Pour ces expériences, nous avons employé des techniques de mesure acoustique. Les expériences ont été menées pour un débit d'air : $Q=1,2 \times 10^{-3} \mathrm{~m}^{3} / \mathrm{s}$, en présence et en absence du déferlement. Ces conditions ont par la suite permis de mieux décrire l'influence du déferlement sur le comportent des bulles d'air. À partir de ces résultats, nous pouvons estimer les caractéristiques hydrodynamiques du rideau de bulles d'air (vitesse du courant d'eau généré par les bulles d'air, carte de l'énergie cinétique des bulles d'air) ainsi que les processus intervenants lors de l'interaction avec le déferlement des vagues tels que le cisaillement et la vorticité. A l'aide de l'ADV nous avons pu mesurer les trois composantes instantanées de la vitesse au niveau de 414 points afin de cartographier le champ des vitesses. La figure 6 présente le champ de vitesse moyenne. Les résultats présentés sur ces cartes permettent de commenter l'effet du déferlement sur le rideau de bulles d'air. La figure 6 illustre l'évolution spatiale du champ de vitesse moyenne des de l'écoulement provoqué par le rideau de bulles sans présence du déferlement. L'écoulement généré par les bulles d'air est bien un écoulement vertical ascendant pour les points de mesures proches du rideau. Cette dynamique ascendante est accompagnée d'une accélération de la vitesse d'écoulement. Près de la surface libre, le courant ascendant change rapidement d'orientation et devient horizontal, d'une manière symétrique par rapport à l'axe du rideau de bulles d'air. Deux tourbillons contra-rotatifs se forment dans cet écoulement. En effet, de part et d'autre de l'axe du rideau de bulle, 


\section{XIII ${ }^{\text {èmes }}$ Journées Nationales Génie Côtier - Génie Civil \\ Dunkerque, 2-4 juillet 2014}

les vecteurs vitesse diminuent en module et s'ajustent de façon à former deux cercles dont les centres se trouvent à $y= \pm 40 \mathrm{~cm}$ et le rayon est sensiblement égal à $30 \mathrm{~cm}$.

Au niveau de la surface libre, le déferlement agit en réorientant le courant vers le fond. Ce processus est enregistré lors du passage de la vague déferlante au cours d'une durée égale à $1 \mathrm{~s}$. De plus, une zone d'interaction entre deux courants est observée. Les deux courants sont générés par le déplacement d'une masse d'eau vers le fond du au déferlement et d'un écoulement opposé généré par l'ascension de bulles d'air vers la surface libre.
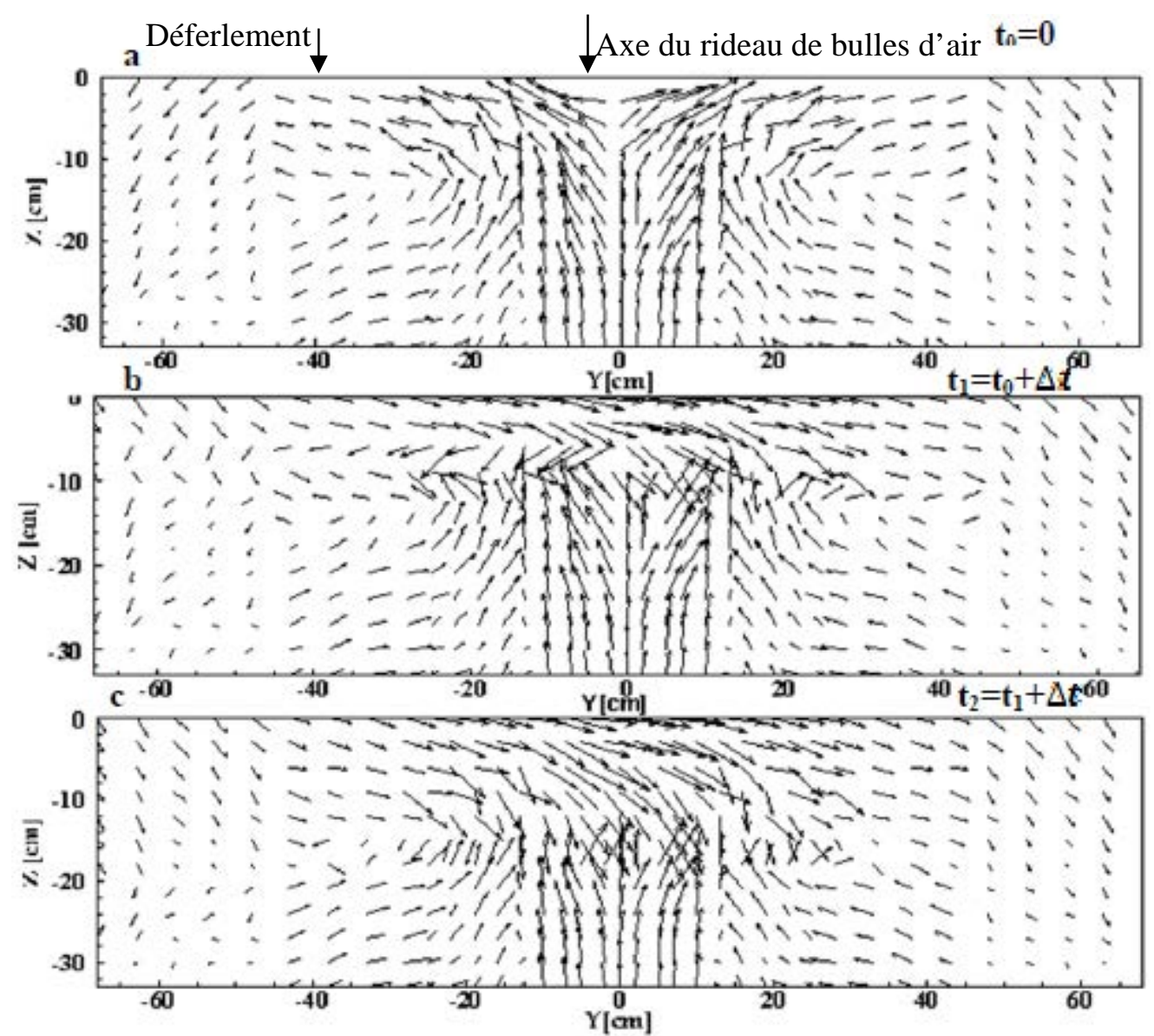

Figure 6. Evolution spatio-temporelle du champ de vitesse avec $Q=1,2 \times 10^{-3} \mathrm{~m}^{3} / \mathrm{s}$ avec le déferlement à trois pas de temps, $\Delta t=0.25 \mathrm{~s}$.

\section{Conclusion}

La finalité de ce travail expérimental est d'améliorer les connaissances actuelles sur les processus physiques qui pilotent les effets de génération de bulles d'air sur le déferlement. L'influence d'un rideau de bulles d'air sur le point de déferlement d'un train d'ondes a été d'avantage appréhendée. Une comparaison des présents résultats avec ceux obtenus par (ZHANG et al., 2010 ; CHANSON \& TOOMBES, 2002) a 
permis de s'assurer de la validité des expériences effectués. Nous avons constaté un déplacement important du point de déferlement vers le générateur des vagues et ce même dans le cas de faibles débits de bulles d'air. Nous avons également montré que le rideau de bulles d'air dissipe une faible quantité de l'énergie des vagues. Les mesures effectuées au moyen de la sonde conductive, en l'absence de vagues, nous ont permis de trouvé que la vitesse des bulles d'air est maximale à la mi-hauteur d'eau.

\section{Références bibliographiques}

AMOL A.K., JYESHTHARAJ B.J. (2005). Bubble Formation and Bubble fuse Velocity in Gas_Liquid Systems: A Review. Eng. Cher,,. Res., Vol. 44, pp 5873_5931.

BRUN-COTTAN J.C. (1986). Vertical transport of particles within the ocean. The role of air-sea exchange in geochemical cycling. NATO ASI Series, Vol. 185, pp 83-111. http://dx.doi.org/10.1007/978-94-009-4738-2_4

BYALKO A.V. (2011). Laminar Bubble Chains: A Logarithmically Exact Solution. Doklady Physics, Vol. 56(2), pp 82-88. http://dx.doi.org/10.1134/S1028335811020121

CHANSON H., TOOMBES L. (2002). Experimental Study of Gas-Liquid Interfacial Properties in a Stepped Cascade Flow. Envi-Fluid-Mech, Vol. 3, pp 241-263. http://dx.doi.org/10.1023/A:1019884101405

CUMMINGS P.D., CHANSON H. (1997). Air entrainment in the developing flow region of plunging jets-Part1: theoretical de development. J. Flui Eng. Vol. 119(3), pp 597-602. http://dx.doi.org/10.1115/1.2819286

GRANT B., DEANE M., DALE S. (1998). Air Entrainment Processes and Bubble Size Distributions in the Surf Zone. Journal of Physical Oceanography, Vol. 29, pp 1393-1403.

KULKARNI A.A., JOSHI J.B. (2005). Bubble Formation and Bubble Rise Velocity in Gas-Liquid Systems: A Review. Ind. Eng. Chem. Res., Vol. 44, pp 5873-5931. http://dx.doi.org/10.1021/ie049131p

MUKHERJEE A., KANDLIKAR S.G. (2007). Numerical study of single bubbles with dynamic contact angle during nucleate pool boiling. International Journal of Heat and Mass Transfer, Vol. 50, pp 127-138. http://dx.doi.org/10.1016/j.ijheatmasstransfer.2006.06.037 VASSILEV A., BEN HADID H., BEN EL HAJEM M., BOTTON V. (2007). Experimental and numerical investigation of an air pocket immersed and immobilized in a horizontal water duct flow. International Journal of Heat and Fluid Flow, Vol. 28, pp 673-682. http://dx.doi.org/10.1016/j.ijheatfluidflow.2007.02.005

WICHTERLE K., SMUTNA K., VECER M. (2009). Shape and rising velocity of bubbles. 36 International Conference of SSCHE Slovikia, Vol. 90, pp 1-12.

ZHANG C., WANG Y., WANG G., YU L. (2010). Wave dissipating performance of air bubble breakwaters with different layouts. Journal of Hydrodynamics, Vol. 22(5), pp 671-680. 